\title{
A CLASS OF INDIRECT UTILITY FUNCTIONS PREDICTING GIFFEN BEHAVIOUR
}

\author{
P.G. MOFFATT, School of Economics, University of East Anglia, UK
}

September 2010

\begin{abstract}
The problem of recognising Giffen behaviour is approached from the standpoint of the Indirect Utility Function (IUF) from which the marshallian demands are easily obtained via Roy's identity. It is shown that, for the two-good situation, downward convergence of the contours of the IUF is necessary for giffenity, and sufficient if this downward convergence is strong enough, in a sense that is geometrically determined. A family of IUFs involving hyperbolic contours convex to the origin, and having this property of (locally) downward convergence is constructed. The marshallian demands are obtained, and the region of Giffen behaviour determined. For this family, such regions exist for each good, and are non-overlapping. Finally, it is shown by geometric construction that the family of Direct Utility Functions having the same pattern of hyperbolic contours also exhibits giffenity in corresponding subregions of the positive quadrant.
\end{abstract}




\section{Introduction}

Ever since Wold \& Jureen's (1953) attempt to illustrate the Giffen paradox by specifying a particular direct utility function, there has been a stream of contributions from authors pursuing similar objectives, for example Spiegel (1994), Weber (1997, 2001), Moffatt (2002) and Sørensen (2006).

One of the lessons learned from this strand of literature is that it is not easy to specify a direct utility function that predicts 'giffen behaviour' and simultaneously satisfies the basic axioms of consumer theory. With this problem in mind, we here adopt an alternative 'dual' approach, starting with an Indirect Utility Function (IUF). The great advantage of using this dual approach is that it is possible for both the IUF and the related marshallian demands to be expressible in explicit closed form.

\section{The Indirect Utility Function (IUF)}

We assume that just two goods, good 1 (price $x$ ) and good 2 (price $y$ ), are available to the consumer. Since we are interested in price effects, we normalise the consumer's income to unity. Let $V(x, y)$ be the consumer's Indirect Utility Function (IUF), i.e. the maximum utility attainable when the prices of the two goods are $x$ and $y$.

The IUF is assumed to satisfy the standard requirements of consumer theory, first, that $V(x, y)$ be monotonic decreasing in both price variables:

$$
V_{x} \equiv \frac{\partial V}{\partial x}<0, \quad V_{y} \equiv \frac{\partial V}{\partial y}<0, \quad \text { in } \quad x>0, y>0
$$

and second, the requirement of 'quasi-convexity', i.e. that the contours of the IUF

be convex to the origin. A clear intuitive proof that quasi-convexity is a necessary property of any IUF is provided by Suen (1992).

\section{Roy's Indentity: a Geometric Interpreta- tion}

Roy's identity is the formula used to obtain marshallian demand functions (i.e. utility maximising quantities expressed in terms of prices) directly from the IUF. Denoting the marshallian demand functions by $g_{1}(x, y)$ and $g_{2}(x, y)$, Roy's identity is:

$$
g_{1}(x, y)=\frac{V_{x}}{x V_{x}+y V_{y}}, \quad g_{2}(x, y)=\frac{V_{y}}{x V_{x}+y V_{y}}
$$


For good 1 (whose price is $x$ ) to be a giffen good, $g_{1}(x, y)$ must be increasing in $x$ in some region, $\Gamma$ say, of the positive $(x, y)$ quadrant. The features required of the IUF for this to occur are most easily seen through the following geometric interpretation of Roy's identity.

Consider an IUF satisfying the asssumptions stated in $\S 1.2$. Let $\mathrm{P}\left(x_{1}, y_{1}\right)$ be a point on any contour $V(x, y)=$ cst. The gradient of the tangent at $\mathrm{P}$ is

$$
d y / d x=-\left[V_{x} / V_{y}\right]_{1},
$$

where the suffix 1 indicates evaluation on at $x=x_{1}, y=y_{1}$. The equation of the tangent at $\mathrm{P}$ is then

$$
y-y_{1}=-\left[V_{x} / V_{y}\right]_{1}\left(x-x_{1}\right),
$$

and this intersects the axis $y=0$ at the point $(h, 0)$, where

$$
h=x_{1}+\frac{y_{1}}{\left[V_{x} / V_{y}\right]_{1}}=\left[\frac{x V_{x}+y V_{y}}{V_{x}}\right]_{1} .
$$

Considering the equations (2), we see that the marshallian demand for good 1 at prices $\left(x_{1}, y_{1}\right)$ is just the reciprocal of $h$ :

$$
g_{1}\left(x_{1}, y_{1}\right)=h^{-1} .
$$

Hence we see that the marshallian demand for good 1 can be interpreted as the reciprocal of the horizontal intercept of the tangent at P. Likewise, the marshallian demand for good 2 is the reciprocal of the vertical intercept of the same tangent.

It follows that the condition required for giffenity of good 1, say, is that the horizontal intercept must move to the left when $x$ increases. That is, for giffenity, $\partial h / \partial x_{1}$ must be negative in some region $\Gamma$ of the $(x, y)$ quadrant. Another way of viewing this requirement is that the tangent gradient (3) must become more negative as $x$ increases, and must do so sufficiently rapidly for neighbouring tangents to intersect above the axis $y=0$. This type of behaviour, involving local downward convergence of adjacent contours, is indicated schematically in figure 1 .

\section{Construction of an Indirect Utility Func- tion (IUF) exhibiting giffenity}

\subsection{Construction of a family of indifference curves in price-space}

We wish to construct a family of contours $V(x, y)=$ cst., having the 'downward convergence' property illustrated in figure 1. To this end, we follow Moffatt(2002) 


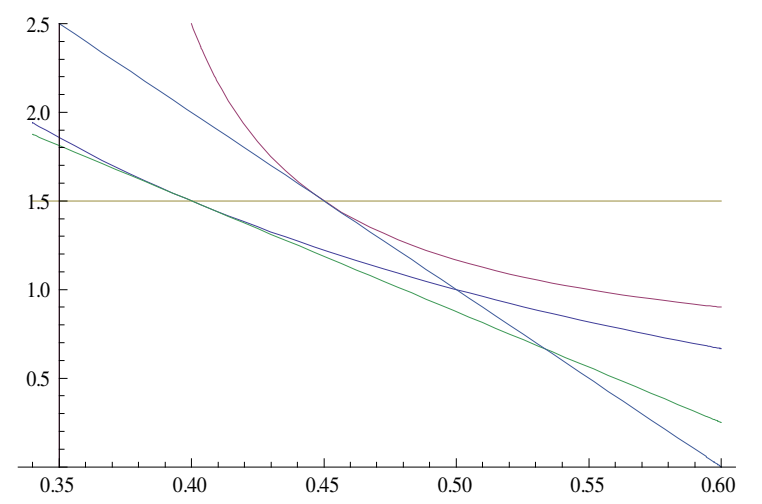

Figure 1: Schematic diagram: the tangents to two adjacent contours at the same value of $y$ intersect above the $y$-axis, a requirement for giffenity of good 1 .

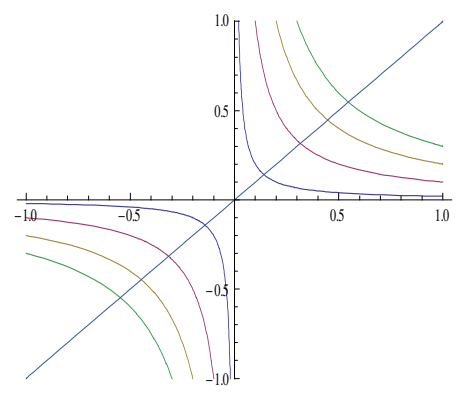

Figure 2: The family of hyperbolæ $x y=c^{2}$.

in constructing a suitable family of hyperbolic contours exhibiting this property in some sub-region of the quadrant $(x>0, y>0)$. First consider the family of hyperbolæ $H: x y=c^{2}$ (figure2). This has two branches:

$$
H^{+}: x y=c^{2}, x>0, y>0, \quad \text { and } \quad H^{-}: x y=c^{2}, x<0, y<0 .
$$

We shall be concerned with just the branch $H^{+}$in what follows. With $y=c^{2} / x$, we have $y^{\prime} \equiv d y / d x=-c^{2} / x^{2}$ and $y^{\prime \prime} \equiv d^{2} y / d x^{2}=2 c^{2} / x^{3}$. So at the point $(c, c)$ on $H^{+}, y^{\prime}=1, y^{\prime \prime}=2 / c$ and the curvature at this point is

$$
\kappa=\frac{y^{\prime \prime}}{\left(1+y^{\prime 2}\right)^{3 / 2}}=\frac{1}{\sqrt{2} c} .
$$




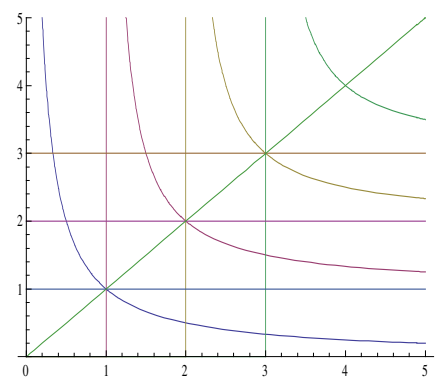

Figure 3: The curves $H_{a}^{+}$(Eqn. 1.9) for $c=1$ and $a=0,1,2,3$. As $a$ increases, the hyperbola is simply shifted out along the diagonal line $y=x$. The asymptotes of the four hyperbolæ are also shown.

Now consider the family of (upper-branch) hyperbolæ(figure 3):

$H_{a}^{+}:(x-a)(y-a)=c^{2}, x>a, y>a$, for variable $a>0$ and fixed $c$.

As $a$ increases, $H_{a}^{+}$simply moves out along the diagonal line $x=y$. Now however, we may achieve the required steepening of the tangents (as in figure 1) by choosing $c$ to be not constant, but rather a positive decreasing function of $a$, i.e. such that $c>0, d c / d a<0$. The hyperbola $H_{a}^{+}$intersects $y=x$ where $(x-a)^{2}=c^{2}$, i.e. where $x=a+c(a)=f(a)$, say, and this function must clearly be monotonic increasing with $a$, in order to ensure that the curves of the family do not intersect; thus we require that

$$
d f / d a=1+d c / d a>0, \quad \text { and so } \quad-1<d c / d a<0 .
$$

The simplest choice is

$$
c(a)=1-\lambda a, \text { where } 0<\lambda<1,
$$

(to satisfy the inequalities $10 p)$. Since $c(a)>0$, we also require that $\lambda a<1$, so this choice of $c(a)$ is limited to the range

$$
0<a<\lambda^{-1}
$$

Figure 4 shows a set of the curves $H_{a}^{+}$with this choice of $c(a)$, which now indicate the downward convergence of the contours above the diagonal that is symptomatic of potential giffenity in good 1 . 


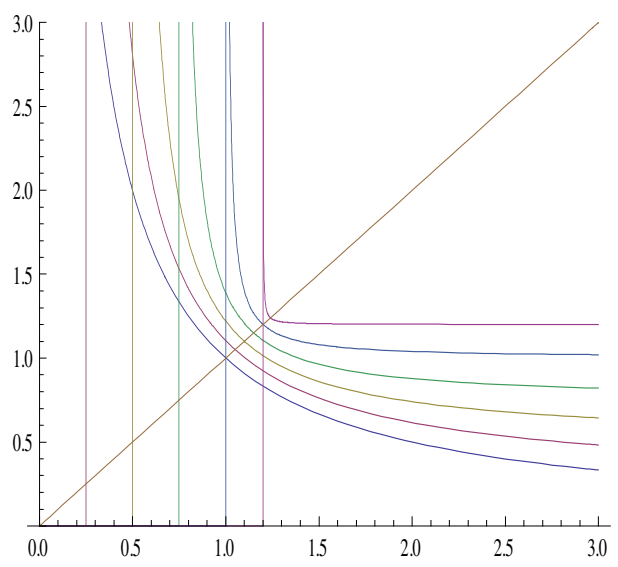

Figure 4: The curves $H_{a}^{+}$(eqn 1.9) for $c=1-\lambda a$, with $\lambda=4 / 5$ and $a=$ $0,0.25,0.5,0.75,1,1.2$. Now, as $a$ increases, the hyperbola is shifted along the diagonal line $y=x$, but with increasing curvature on this diagonal. The convergence of the hyperbolæ in the region above the diagonal is an indication of potential giffenity.

\subsection{Further considerations}

Actually (11) is not ideal as a choice of $c(a)$, because it would require that we match smoothly to a different function to cover the region below the curve $x y=1$. We can circumvent this by simply shifting the curves of figure 4 down the diagonal until this lowest member passes through the origin. This is achieved by the choice 2

$$
H_{a}^{+}:(x-a)(y-a)=c^{2}, \text { for variable } a \geq-1, \text { and } c(a)=1-\lambda-\lambda a,
$$

with $0<\lambda<1$. Note that when $a=-1, c(-1)=1$, and the corresponding hyperbola $(x+1)(y+1)=1$ has 'centre' at $(-1,-1)$ and passes through $(0,0)$. Also, although the downward convergence of contours can be expected only for $c(a)>0$, i.e. for $a<(1-\lambda) / \lambda$, we may in fact adopt the form (1.13) also for $a>(1-\lambda) / \lambda$, where the curvature on $x=y$ is then $-(\sqrt{2} c(a))^{-1}$ and decreases with increasing $a$. These properties are evident in figure 5, which shows this family of hyperbolæ, for the choice $\lambda=4 / 5$. For this value, the point of maximum curvature is $(\mu, \mu)$, where $\mu \equiv(1-\lambda) / \lambda=1 / 4$, so for $a<1 / 4$, the curves are

\footnotetext{
${ }^{2}$ The vanishing of $c$ for $a=(1-\lambda) / \lambda$ means that the particular contour $a=(1-\lambda) / \lambda$ has a rightangled corner at the point $(\mu, \mu)$, where $\mu=(1-\lambda) / \lambda$. This singularity may be removed by adopting a modified $c(a)$ such that $c^{2}=(1-\lambda-\lambda a)^{2}+\epsilon^{2}$, for suitably small $\epsilon$. We shall not pursue this refinement here.
} 


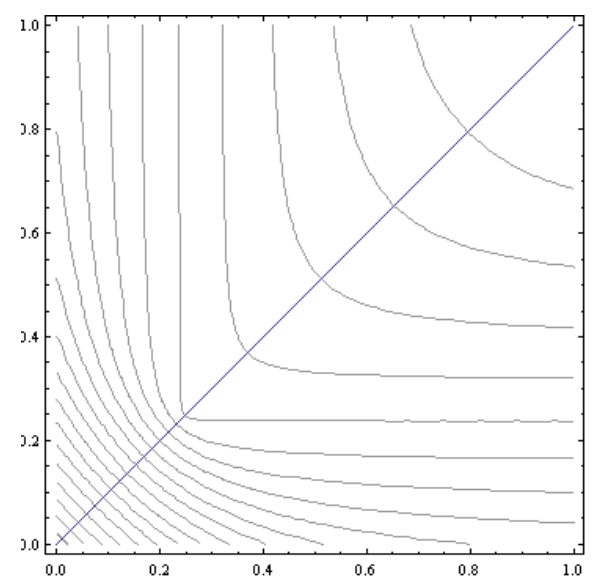

Figure 5: The family of curves $H_{a}^{+}$defined by eqn (1.13), for the choice $\lambda=4 / 5$. The curve $a=1 / 4$ (not shown in the figure) exhibits a corner at the point $(1 / 4,1 / 4)$.

converging downwards (above the diagonal line $x=y$ ), and we may anticipate giffen behaviour in a region above this diagonal near the point $(1 / 4,1 / 4)$, where this downward convergence is strong. For $a>1 / 4$, the contours show downward divergence above the diagonal, and giffenity cannot occur here.

Let $(X, Y)$ be a point on $H_{a}^{+}:(x-a)(y-a)=c(a)^{2}$, so that

$$
X=a+c(a)^{2} /(Y-a) .
$$

The gradient of $H_{a}^{+}$at $(X, Y)$ is

$$
\left[\frac{d y}{d x}\right]_{(X, Y)}=-\frac{c(a)^{2}}{(X-a)^{2}}=-\frac{(Y-a)^{2}}{c(a)^{2}}
$$

so the tangent to $H_{a}^{+}$at $(X, Y)$ has equation

$$
y-Y=-(Y-a)^{2}(x-X) / c(a)^{2} .
$$

This cuts the axis $y=0$ at the point $(h, 0)$, where $(\mathrm{cf}(5))$

$$
h=X+\frac{c(a)^{2} Y}{(Y-a)^{2}}=a+\frac{c(a)^{2}}{Y-a}+\frac{c(a)^{2} Y}{(Y-a)^{2}},
$$

which simplifies to

$$
h(a, Y)=a+\frac{c(a)^{2}(2 Y-a)}{(Y-a)^{2}} .
$$




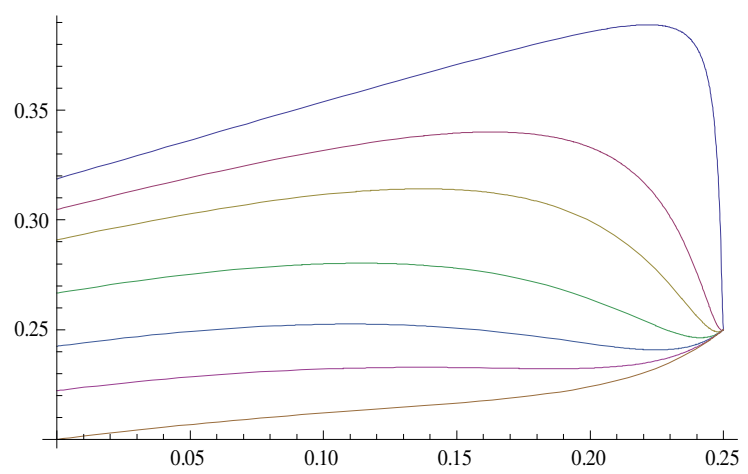

Figure 6: The function $h(a, Y)$ for $\lambda=4 / 5$, and $Y=$ $0.251,0.2625,0.275,0.3,0.33,0.36,0.4$ (values increasing downwards). The uppermost five curves all show regions of negative gradient; this is the region of potential giffenity.

Figure 6 shows $h(a, Y)$ as a function of $a$ with $c(a)=1-\lambda-\lambda a$, for the choice $\lambda=4 / 5$ and for seven values of $Y$ greater than $(1-\lambda) / \lambda(=1 / 4)$. A range of negative gradient is apparent on the curves for which $0.25<Y<0.36$.

Now we are interested in the variation of $h$ with $X$ at constant $Y$, i.e. in

$$
\partial h(a, Y) / \partial X=(\partial h(a, Y) / \partial a)(\partial a / \partial X)
$$

It is obvious that, at constant $Y, a$ is an increasing function of $X$, i.e. $\partial a / \partial X>0$. So the sign of $\partial h(a, Y) / \partial X$ is the same as that of $\partial h(a, Y) / \partial a$. The contour in the $(a, Y)$-plane on which $\partial h(a, Y) / \partial a$ is zero is shown in figure 7 (again for the choice $\lambda=4 / 5)$; inside this contour, $\partial h(a, Y) / \partial a$ is negative, and this is the region of potential giffenity.

\subsection{The IUF in explicit form}

Now we are in a position to obtain an explicit form for the IUF exhibiting giffenity. First note that for each $a$ we have a unique upper-branch hyperbola given by

$$
H_{a}^{+}:(x-a)(y-a)=(1-\lambda-\lambda a)^{2} .
$$

Thus, $a$ can be regarded as a label, constant on each $H_{a}^{+}$. Conversely, the member of the family $H_{a}^{+}$passing through any given point $(x, y)$ is labeled by a root of 


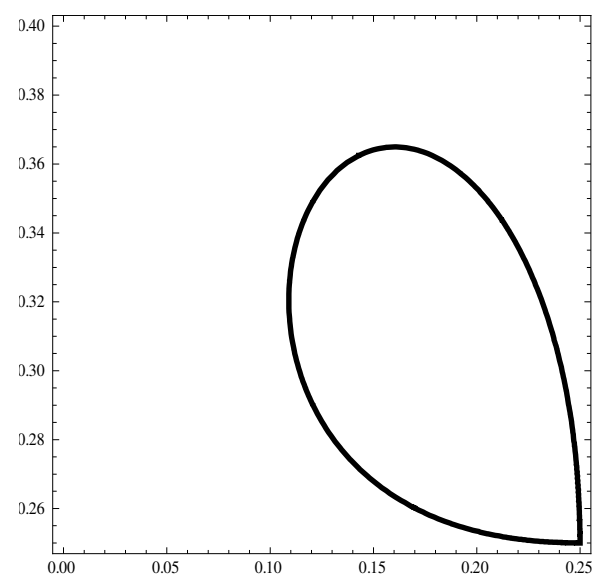

Figure 7: Contour $\partial h / \partial a=0$ in the $(a, Y)$-plane $(\lambda=4 / 5,0<a<1 / 4,1 / 4<Y<$ $2 / 5) ; \partial h / \partial a$ is negative inside this critical contour. This is the region $\Gamma$ referred to in $\S 1.3$.

(20) regarded as a quadratic equation for $a$. The relevant root (which vanishes as it should when $\left.x y=(1-\lambda)^{2}\right)$ is

$$
a=\frac{x+y-2 \lambda(1-\lambda)-S(x, y)}{2\left(1-\lambda^{2}\right)},
$$

where

$$
S(x, y)=\sqrt{[x+y-2 \lambda(1-\lambda)]^{2}-4\left(1-\lambda^{2}\right)\left[x y-(1-\lambda)^{2}\right]} .
$$

The other root (with $-S$ replaced by $+S$ ) corresponds to the lower branch $H_{a}^{-}$, and can be ignored. The contours $a(x, y)=$ cst. should be effectively the same as those in figure 5. Figure 8 show these contours for two values of $\lambda$ plotted directly from (21). Note how, as $\lambda$ increases towards unity, the point of maximum curvature $(\mu, \mu)$, where $\mu=(1-\lambda) / \lambda$, moves towards the origin, and the downward 'giffenitypromoting' convergence of the contours above the diagonal $x=y$ increases; this trend persists as $\lambda$ increases further towards unity.

The function $a(x, y)$ is clearly an increasing function of $x$ and of $y$, i.e. $\partial a / \partial x>$ $0, \partial a / \partial y>0$. The IUF must, of course, be decreasing in $x$ and $y$. Accordingly, let $V(a)=-a$, giving rise to the following IUF:

$$
V(x, y)=-\frac{x+y-2 \lambda(1-\lambda)-S(x, y)}{2\left(1-\lambda^{2}\right)},
$$




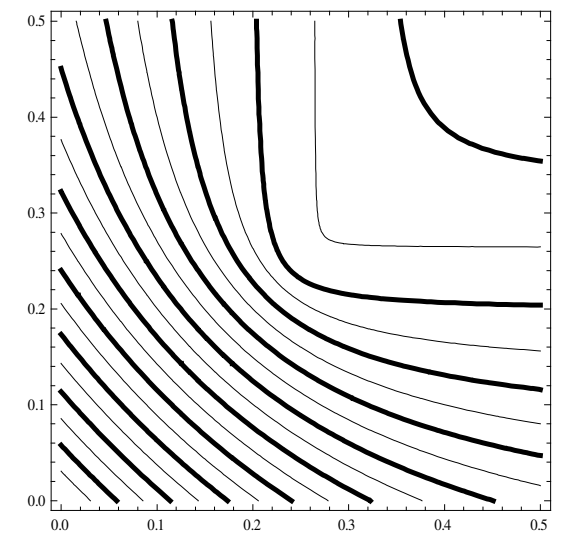

(a)

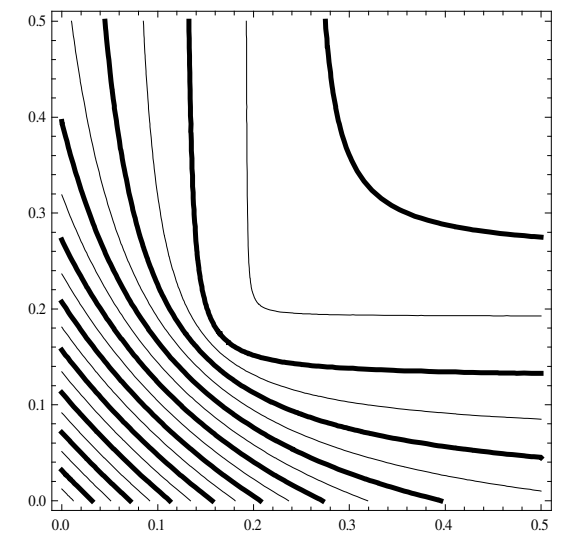

(b)

Figure 8: Contours $a(x, y)=$ cst., plotted directly from the function (1.21). (a) $\lambda=0.8$, $\mu(=(1-\lambda) / \lambda)=0.25$; (b) $\lambda=0.85, \mu=3 / 17=0.176 \ldots$. Note how the critical ('corner') point $(\mu, \mu)$ moves towards the origin, and the downward convergence of the contours above the diagonal line $x=y$ increases (for $y<\mu$ ), with increase of $\lambda$, a trend that persists as $\lambda$ increases towards unity. 


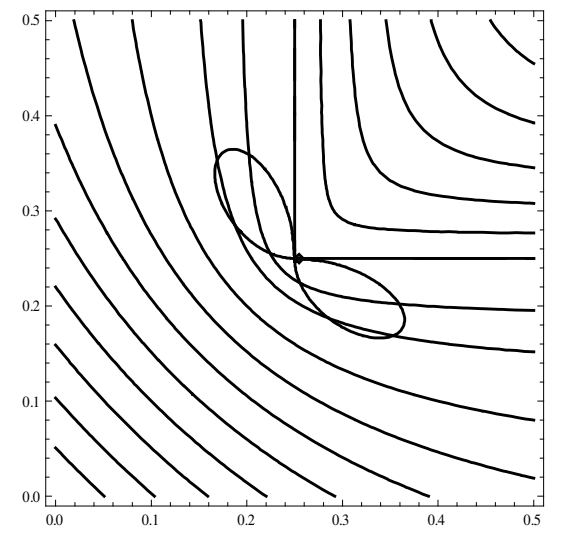

Figure 9: Contours of the IUF given by equation (23) (with $\lambda=4 / 5$ ) and (superposed) the region $\Gamma$ of giffenity of good 1 (above the diagonal); by symmetry, the region of giffenity of the good 2 is obtained, as shown, by simple reflection in the diagonal $x=y$.

where $S(x, y)$ is as defined in 22 . 23) is an IUF with the desired properties, i.e. being continuous and quasi-convex over the whole quadrant, satisfying the inequalities (1), and exhibiting the property of giffenity (in good 1) in the region indicated by figure 7

The contours of this Indirect Utility Function $V(x, y)$ are shown in figure 9 , again for the representative choice $\lambda=4 / 5$; and, superposed on this, the closed contour (above the diagonal) within which the giffenity property is satisfied (obtained by substituting (21) in the expression for $\partial h(a, Y) / \partial a$ obtained by differentiating (18)). This is the region $\Gamma$ referred to in $\S 1.3$. Note that, by the symmetry of the IUF about the diagonal, good 2 also exhibits giffenity in the similar region that is the mirror image of $\Gamma$ in the diagonal, as shown in the figure.

\subsection{Marshallian Demands}

Armed with an IUF in explicit form, we are now in a position to calculate the marshallian demands via Roy's identity (2). The derivatives $V_{x}$ and $V_{y}$ can be obtained by straightforward differentiation of the above expression (23) for $V(x, y)$, and $g_{1}$ and $g_{2}$ may then be constructed from (2), and simplified. The Marshallian demand for good 1 so obtained is

$$
g_{1}(x, y)=\frac{-x+y\left(1-2 \lambda^{2}\right)+2 \lambda(1-\lambda)+R(x, y)}{(x+y)[2 \lambda(1-\lambda)-R(x, y)]-(x-y)^{2}},
$$




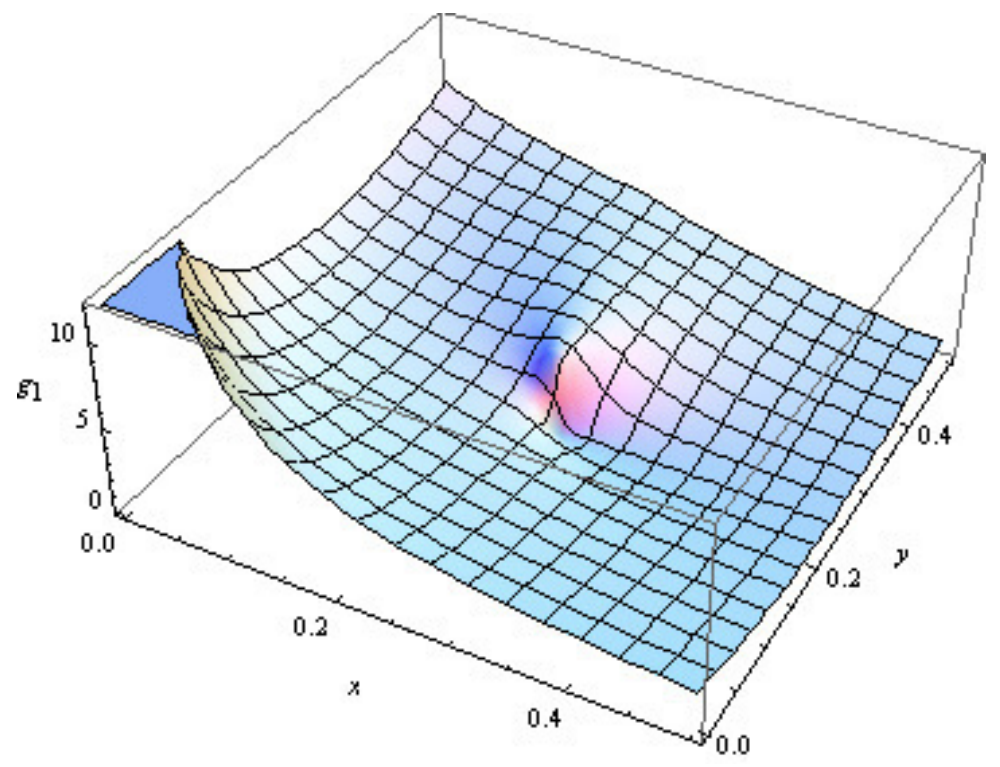

Figure 10: A 3D Plot of the the Marshallian demand function $g_{1}(x, y)$, obtained from (23) and (2) with $\lambda=4 / 5$.

where

$$
R(x, y)=\sqrt{x^{2}+y^{2}+4(\lambda-1)\left[\lambda-1+\lambda(x+y)+2 x y\left(2 \lambda^{2}-1\right)\right]} .
$$

The Marshallian demand function (24) may be represented graphically in a variety of ways. First of all, a $3 \mathrm{D}$ plot (with $\lambda=4 / 5$ ), is shown in figure 10 . There is clearly a region near the centre of this plot in which the function appears to increase as $x$ increases - this is the "giffen region". The position of this region may be more precisely identified my means of a contour plot. The contours $g_{1}(x, y)=$ cst. are shown in figure 11 (again with $\lambda=4 / 5$ ), the values of $g_{1}$ on the various contours being as indicated; only the interesting region near the point $(1 / 4,1 / 4)$ is shown, and the curve bounding the "giffen region" within which $\partial g_{1} / \partial x$ is positive is superposed.

Finally, we may plot the Marshallian demand for good 1 against the price of good 1 , for a fixed price of good 2. Such a plot is shown in 12, for which the price of good 2 has been set to $y=0.30$. This plot clearly shows that when the price of good 1 is between 0.18 and 0.24 , the function is positively sloped, confirming giffenity beyond any doubt.

In this section we have focused attention on the Marshallian demand for good 1. However, note that the symmetry of $V(x, y)$ implies that $g_{2}(x, y) \equiv g_{1}(y, x)$, so 


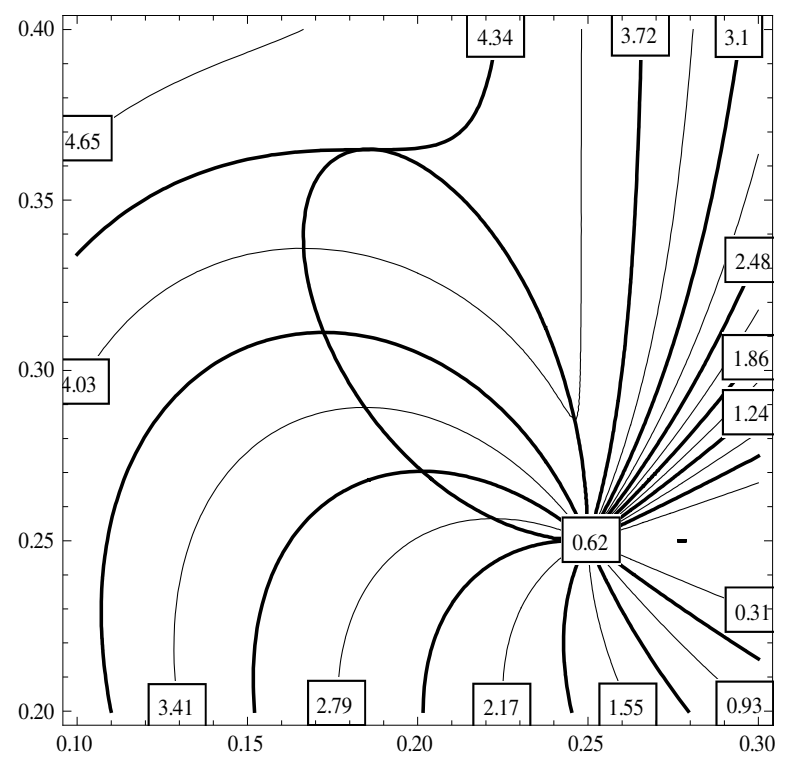

Figure 11: Contours of $g_{1}(x, y)$ in the region $(0.1<x<0.3,0.2<y<0.4)$, obtained from (23) and (2) with $\lambda=4 / 5$. The curve bounding the giffen region $\Gamma$, within which $\partial g_{1} / \partial x>0$, is superposed; note that the tangents to the contours are horizontal on this curve.

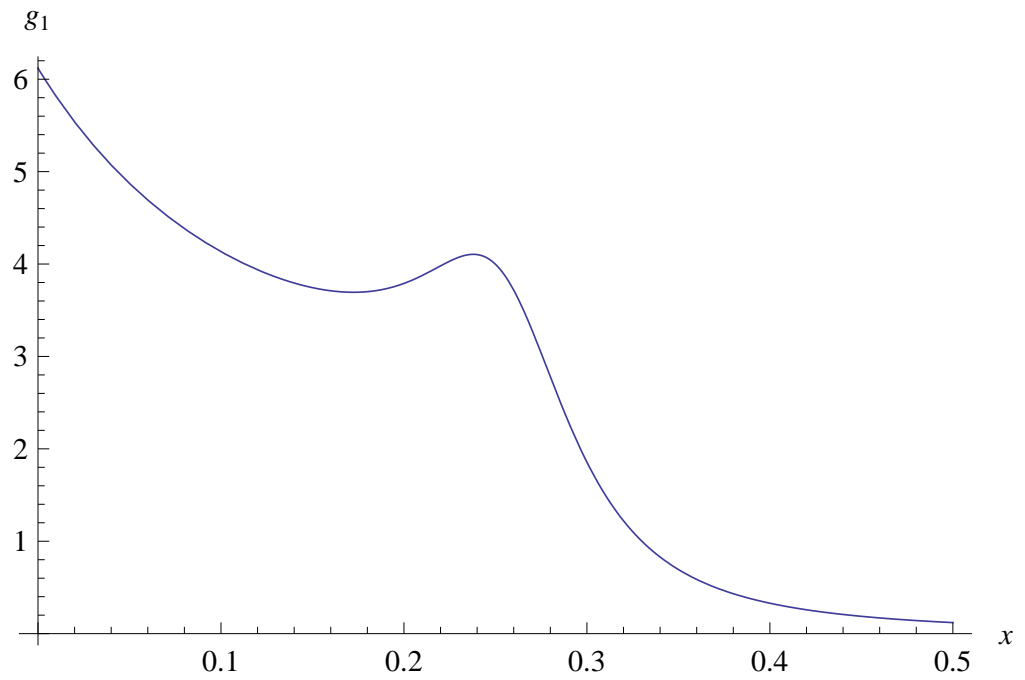

Figure 12: A plot of the the Marshallian demand function $g_{1}(x, y)$ against $x$, with $\lambda=4 / 5$ and $y$ set to 0.30 . 
exactly the same patterns would be observed for good 2 .

\section{A Direct Utility Function exhibiting giff- enity}

It is interesting that the class of function developed in $\S 1.4$ for the IUF can also be used to construct a direct utility function that can predict giffenity. In this Section, let us therefore redefine $x$ and $y$ as the quantities consumed of the two goods and consider the direct utility function $U(x, y)$.

Recall that in (21), we have a function that is increasing in $x$ and $y$, and it was this function to which we applied a sign reversal in order to obtain our IUF (23). Let us now use (21) itself as the direct utility function:

$$
U(x, y)=\frac{x+y-2 \lambda(1-\lambda)-S(x, y)}{2\left(1-\lambda^{2}\right)},
$$

where $S(x, y)$ is still as defined in (22). Clearly, for any given $\lambda,(1.25)$ has an indifference map which is identical to that of the IUF (23).

In figure 13 , we plot the contours of (26) with $\lambda=0.9$ (the corner singularity being now at $(1-\lambda) / \lambda=1 / 9)$, and two budget lines which show the effect of a rise in the price of good $x$. The upper budget line applies before, and the lower one after, the price rise. The important point is that the horizontal position of the point of tangency is further to the right following the price rise, implying that $x$ is a giffen good at the assumed price combination. Since $(26)$ is symmetric in $x$ and $y$, price combinations can also be chosen for which good $y$ is giffen.

In (Moffatt \& Moffatt 2010), it is proved that the phenomenon demonstrated above is symptomatic of a general result: namely, that any two-good IUF that exhibits giffenity in one of the goods can be transformed, simply by reversing the sign, into a direct utility function that exhibits giffenity in the other good.

\section{Conclusion}

The purpose of this paper has been to construct an Indirect Utility Function (IUF) that predicts giffenity. One considerable advantage from this approach is that, given that such a function can be found, Roy's identity can be used to obtain the marshallian demands explicitly.

A family of such IUFs has indeed been found, dependent on the parameter $\lambda$ $(0<\lambda<1)$. The key feature of this family is that the maximum curvature of each contour increases as we move out from the origin. An economic interpretation of 


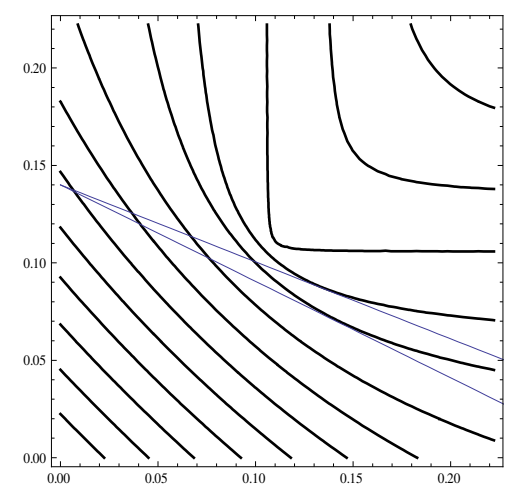

Figure 13: The direct utility function 26 with $\lambda=0.9$. The two budget lines represent the positions before and after a rise in the price of good $x$. The lower budget line applies after the price rise. It is seen that the horizontal position of the point of tangency is further to the right following the price rise.

this feature would be that as purchasing power rises, the two goods become closer complements, and less close substitutes.

An example from this class of function (for the choice $\lambda=0.8$ ) has been presented graphically in figure 9. Because of the symmetry of the function, both of the goods are giffen goods, and the sets of price combinations over which giffenity applies to each good are also shown in this figure. These two sets are non-overlapping.

The Marshallian demand function corresponding to the IUF represented in figure 9 has then been derived using Roy's identity, and presented algebraically in (24). This function has then been presented graphically in a variety of ways, all of which confirm the existence of a region of giffenity.

The chosen class of functions can also be used to provide Direct Utility Functions, and, as demonstrated in figure 13 (for the choice $\lambda=0.9$ ), such an approach can also provide a demonstration of giffenity. However, it is not in general possible to derive explicit expressions for the marshallian demands starting from such a Direct Utility Function. The fact that this is, by contrast, straighforward when starting from the IUF provides the rationale for the approach adopted in this Paper. 


\section{References}

[1] Moffatt P. G. 2002. Is Giffen behaviour compatible with the axioms of consumer theory? Journal of Mathematical Economics 37:259-267

[2] Moffatt, P.G., Moffatt, H.K. 2010. Giffen Goods: a Duality Theorem. Mimeo, Univ. of East Anglia.

[3] Sorenson P. 2006. Simple Utility Functions with Giffen Demand Economic Theory 31:367-70

[4] Spiegel U. 1994. The Case of a Giffen Good. Journal of Economic Education 25:137-147

[5] Suen W. 1992. A diagrammatic proof that indirect utility functions are quasiconvex Journal of Economic Education 23:53-55

[6] Weber C.E. 1997. The case of a Giffen Good: Comment Journal of Economic Education 28:36-44

[7] Weber C.E. 2001. A Production Function with an Inferior Input The Manchester School 69:616-622

[8] Wold H., Jureen L. 1953. Demand Analysis New York: Wiley. 